\title{
Determination of an Optimal Frailty Cutoff Score of Tilburg Frailty Indicator and Frailty Associated Factors in Community-Dwelling Turkish Older Adults
}

\author{
Gulsah Ozsoy ${ }^{1}$, Esra Ates Bulut ${ }^{2}$, Baris Gurpinar ${ }^{3}$, Nursen Ilcin ${ }^{3}$, Ahmet Turan Isik ${ }^{4}$ \\ ${ }^{1}$ Department of Physiotherapy and Rehabilitation, Faculty of Health Sciences, Selcuk University, Konya, Turkey \\ ${ }^{2}$ Department of Geriatric Medicine, Adana City Training and Research Hospital, Adana, Turkey \\ ${ }^{3}$ School of Physical Therapy and Rehabilitation, Dokuz Eylul University, Izmir, Turkey \\ ${ }^{4}$ Department of Geriatric Medicine, Faculty of Medicine, Dokuz Eylul University, Izmir, Turkey
}

\section{Corresponding Author:}

Gulsah Ozsoy, $\mathrm{PhD}$

Department of Physiotherapy and

Rehabilitation, Faculty of Health

Sciences, Selcuk University, Ardicli,

Celal Bayar Cd. No:299, 42250

Selcuklu/Konya, Turkey

E-mail: fzt.gulsah@hotmail.com

ORCID:

https://orcid.org/0000-0001-5678-771X

Received: August 1, 2021

Revised: November 4, 2021

Accepted: November 6, 2021

\begin{abstract}
Background: Frailty is a multidimensional and dynamic state that has adverse physical, psychological, and social outcomes. The Tilburg Frailty Indicator (TFI) has the most robust evidence of reliability and validity for assessing frailty. However, the characteristics of TFI have not been investigated in detail. This study aimed to set a cutoff score for frailty and evaluate frailty-associated factors in community-dwelling older adults. Methods: This cross-sectional study assessed frailty according to both the TFI and Fried criteria. The Geriatric Depression Scale, basic and instrumental activities of daily living, and Hospital Anxiety and Depression Scale-Anxiety subscale were also implemented. Results: This study included 166 older adults. The area under the receiver operating characteristic curve was 0.735 (95\% confidence interval, 0.648-0.823). A TFI cutoff point of 8 , showed a sensitivity of $60 \%$ and specificity of $72.5 \%$ for the prediction of frailty $(p<0.05)$. Frailty according to the TFI was more associated with the physical and psychological parameters, while frailty according to the Fried score was more closely related to the physical parameters $(p<0.05)$. Conclusion: The results of this study suggested an optimal TFI cutoff score of 8 as a frailty instrument in community-dwelling older adults. Additionally, the TFI included physical, psychological, and social aspects, thereby providing a multidimensional evaluation of frailty.
\end{abstract}

Key Words: Aged, Frailty, Tilburg Frailty Indicator

\section{INTRODUCTION}

Frailty is an important geriatric syndrome characterized by an age-associated decline in physiologic reserve that causes serious adverse health consequences such as hospitalization, institutionalization, and mortality. ${ }^{1)}$ Frail persons have increased risks of vulnerability and death due to minor external stress. Frailty is a multidimensional and dynamic state with adverse physical, psychological, and social outcomes. ${ }^{2)}$

Although frailty is an important concern worldwide, no international standard definition of frailty has been established. ${ }^{3)}$ In recent years, healthcare professionals and researchers have suggested the need to evaluate frailty using a multidimensional approach that includes physical, psychological, and social dimensions. ${ }^{4}$ The commonly used frailty scales such as the Fried Frailty Index ${ }^{5)}$ and FRAIL $^{6)}$ do not evaluate the psychosocial aspects of the patients. A systematic review demonstrated that the Tilburg Frailty Indicator (TFI) has the most robust evidence of reliability and validity for assessing frailty in older adults among 38 multi-component frailty assessment tools. ${ }^{7)}$ The TFI is a multidimensional, useful, and applicable instrument that assesses the physical, psychological, and social components of frailty in older adults. ${ }^{8)}$ The TFI has 
been translated and validated in many languages. ${ }^{9,10)}$

Different studies have reported different TFI cutoff scores. A previous study showed that a total score of 5 or more was the optimal cutoff for assessing frailty in Dutch persons who were aged 75 years and older, ${ }^{8)}$ while a TFL cutoff score of 6 was reported in Portuguese individuals (aged $\geq 65$ years) ${ }^{11)}$ and a cutoff score of 5 was reported among Chinese community-dwelling older adults (aged $\geq 60$ years). ${ }^{9)}$ Thus, different cutoff scores have been identified for different populations. In addition, the characteristics of the TFI have not been investigated in detail. Especially, the relationship between the TFI and sarcopenia according to the revised European Working Group on Sarcopenia in Older People (EWGSOP-2) requires investigation and the risk factors for frailty should be determined. Therefore, this study aimed to determine a TFI cutoff value and frailty risk factors in older adults.

\section{MATERIALS AND METHODS}

\section{Study Design and Participants}

This cross-sectional study included 166 participants who were aged 60 years or older and admitted to the geriatric outpatient clinic in Turkey. We excluded individuals with severe illnesses that affected their general health statuses, such as congestive heart failure (Class III and IV), respiratory failure, acute coronary syndrome, acute renal failure, and cancer. Since the reliability of handgrip strength is low in older patients with dementia because of their difficulty in comprehension, which can cause them to fail to complete tasks, we also excluded patients with moderate and severe dementia. $^{12)}$

All procedures performed in studies involving human participants or human tissue were in accordance with the ethical standards of the Institutional Ethical Review Board of Dokuz Eylul University (No. 3282-GOA) and with the 1975 Declaration of Helsinki and its later amendments or comparable ethical standards. Informed consent was obtained from all participants included in the study.

\section{Data Collection}

\section{Participant characteristics}

Participant characteristics (age, sex, years of education, comorbidities, falls in the previous year, and the number of medications) were recorded. On admission, the participants were asked whether they had fallen in the previous year. Dementia and depression were diagnosed according to the Diagnostic and Statistical Manual of Mental Disorders 5th Edition (DSM-5) criteria. ${ }^{13)}$ A comprehensive geriatric assessment was performed for each participant, in- cluding the Mini-Mental State Examination (MMSE), ${ }^{14)}$ Clinical Dementia Rating Score (CDR), Geriatric Depression Scale (GDS), ${ }^{15)}$ basic and instrumental activities of daily living (BADL, IADL), ${ }^{16)}$ Tinetti Performance-Oriented Assessment of Mobility (POMA), ${ }^{17)}$ and Mini Nutritional Assessment (MNA). ${ }^{18)}$ Additionally, the Hospital Anxiety and Depression Scale-Anxiety subscale (HADS-A) was used to assess anxiety. The HADS-A comprises seven items, each of which is scored from 0 (not present) to 3 (considerable). ${ }^{19)}$

\section{Laboratory measurements}

Laboratory tests, such as renal and liver functions and fasting blood glucose, hemogram, thyroid-stimulating hormone (TSH), C-reactive protein (CRP), vitamin D, vitamin B12, and folic acid levels were measured to evaluate the biochemical, metabolic, and nutritional status of the patients. These values were all obtained using an auto-analyzer diagnostic modular system (Roche E170 and P800; Roche, Basel, Switzerland). Serum 25-hydroxyvitamin $\mathrm{D}(25(\mathrm{OH}) \mathrm{D})$ levels were measured by radioimmunoassay.

\section{Frailty assessment}

We assessed frailty according to the TFI and modified Fried Physical Frailty Scale. The TFI has two parts. Part A contains 10 items with different predictors of frailty based on socio-demographic data, while Part B contains 15 total items to assess physical ( 8 items), psychological (4 items), and social (3 items) parameters. The total score of the scale is calculated from Part B, with high scores indicating increased frailty. ${ }^{8)}$ Frailty was also evaluated using the modified Fried Physical Frailty Scale. According to this scale, the presence of three or more of the following five criteria indicated frailty: weight loss, exhaustion, low physical activity, slowness, and weakness.

\section{Sarcopenia assessment}

We assessed sarcopenia according to the revised version of the EWGSOP, which recommends evaluating muscle strength, muscle quantity, and physical performance while considering ethnic differences. ${ }^{20)}$ Therefore, the cutoff points for skeletal muscle mass and strength were accepted according to those reported in a validated Turkish study. ${ }^{21)}$

Handgrip strength was measured using a JAMAR hydraulic hand dynamometer (Model J00105; Lafayette Instrument, Lafayette, IN, USA). The arm was positioned at the side of the body, and the dynamometer was held with the elbow flexed to $90^{\circ}$. The measurements were repeated three times, with the maximum value of the dominant hand used in the analyses. Low muscle strength (MS) was defined as $<14 \mathrm{~kg}$ and $<28 \mathrm{~kg}$ in women and men, re- 
spectively. $^{21)}$

Bioelectrical impedance analysis (BIA) was performed using a TANITA device (MC-780U, multi-frequency segmental body composition analyzer; TANITA, Tokyo, Japan). Based on the muscle mass bioimpedance values, skeletal muscle (SM, in kg) was calculated as: ${ }^{22)}$

$\left[\left(\right.\right.$ height $\left.\left.^{2} / \mathrm{R}\right) \times 0.401\right]+($ gender $\times 3.825)+($ age $\times-0.071)+$ 5.102

The skeletal muscle mass index (SMI) was calculated by dividing the skeletal muscle mass (SMM) in kilograms by the length in square meters $\left(\mathrm{SMI}=\mathrm{SMM} /\right.$ height $\left.^{2}\right)$. Low muscle quantity was defined as $<5.70 \mathrm{~kg} / \mathrm{m}^{2}$ and $<8.33 \mathrm{~kg} / \mathrm{m}^{2}$ in female and male, respectively. ${ }^{21)}$

We used the 4-m walking time to assess physical performance, with low physical performance defined as a rate of $\leq 0.8 \mathrm{~m} / \mathrm{s}^{20}$ )

\section{Sample Size}

The minimum sample size was calculated using the Power Analysis and Sample Size (PASS) software version 11 (NCSS Software, Kaysville, UT, USA). ${ }^{23)}$ Assuming a prevalence of frailty of $28 \%$, ${ }^{12)}$ at least 150 patients were required (prevalence $=28 \%$, hypothesis null $=0.6$, hypothesis alternative $=0.8$, power of $80 \%$ ).

\section{Data Analysis}

We performed all statistical analyses in SPSS Statistics for Windows version 22.0 (IBM Corp., Armonk, NY, USA). We used Shapiro-Wilk test and histograms to check for the normality of the data distributions. Mean and standard deviation (SD) values were reported for continuous variables and percentage values for categorical variables.

We assessed the areas under curves of receiver operating characteristic (ROC) analysis to test the predictive accuracy of the TFI for the identification of patients with frailty according to the Fried Frailty Index, which is considered the "gold standard" for evaluating frailty, ${ }^{5,24)}$ and to set an appropriate cutoff point. We considered test values of $<0.7,0.7-0.9$, and $>0.9$ to indicate low moderate (useful for some purposes), and high accuracy, respectively. ${ }^{25)}$

We performed binary logistic regression analysis to identify variables that were potential risk factors (independent variables: POMA total, POMA gait, POMA balance, walking speed, BADL, HADS-A, GDS, IADL, MNA, low MS, low SM, sarcopenia) of frailty according to the TFI and the Fried Frailty Index (dependent variable: persons with TFI $<8$ and Fried $<3$ who were not frail). The regression analysis included continuous variables (total POMA gait, POMA balance, walking speed, BADL, HADS-A, GDS, IADL, and MNA) and categorical variables (low MS, low $\mathrm{SM}$, and sarcopenia). The reference group notations for low MS, low SM, and sarcopenia were 1 . We expressed the results as adjusted odds ratios (ORs) with corresponding 95\% confidence intervals (CIs). The level of significance was set at $\mathrm{p}<0.05$.

\section{RESULTS}

This study included a total of 166 older adult patients. The mean ages in the frail and non-frail groups were 73.10 years and 72.89 years, respectively (Table 1). The characteristics and outcome measures of the participants are presented in Table 1. The non-frail group showed higher education level, laboratory values (hemoglobin, vitamin D, and vitamin B12 levels), POMA score (total, balance, gait), activities of daily living (IADL and BADL scores), MNA score, handgrip strength, SMM, SMI, gait speed, and BMR and lower number of medications, falls, GDS, and HADS-A scores, than the frail group $(\mathrm{p}<0.05)$ (Table 1$)$. The Fried frailty states differed between groups $(\mathrm{p}<0.05)$ (Table 1, Fig. 1). The comorbidities were similar between the groups $(p>0.05)$ (Table 1$)$.

The area under the ROC curve was 0.735 (95\% CI, 0.648 0.823 ). A TFI cutoff point of 8 , showed a sensitivity of $60 \%$ and specificity of $72.5 \%$ for the prediction of frailty $(p<0.05)$. In the assessment of the diagnostic value of the area under the curve, this value was significant $(\mathrm{p}<0.05)$ (Fig. 2).

Frailty according to the TFI was more associated with lower POMA total-balance scores, lower BADL scores, higher HADS-A scores, higher GDS scores, lower MNA scores, and low MS. Frailty according to the Fried score was more closely related to lower POMA total-balance scores, lower walking speed, lower ADL scores, lower MNA score, and low MS ( $\mathrm{p}<0.05)$ (Table 2). Neither TFI nor Fried scores were associated with sarcopenia $(\mathrm{p}>0.05)$ (Table 2).

\section{DISCUSSION}

The main finding of the study was that a total TFI score of 8 or more was the optimal cutoff for assessing frailty in older adults. Second, the Fried score was more closely related to physical parameters, while the TFI score was more associated with both psychosocial and physical parameters. Third, the measures of psychosocial and physical parameters were better in the non-frail group than those in the frail group. Additionally, neither the TFI nor the Fried scores were associated with sarcopenia.

Frailty is one of the most crucial problems associated with a high risk of adverse outcomes. Frailty is a multidimensional and dynamic state that has adverse physical, psychological, and social outcomes. ${ }^{2)}$ The TFI had the most robust evidence of reliability and validity for assessing frailty in older adults among 38 multi-compo- 
Table 1. Participant characteristics

\begin{tabular}{|c|c|c|c|c|}
\hline & All participants $(n=166)$ & Frail group $($ TFI $\geq 8)(n=57)$ & Non-frail group $(\mathrm{TFI}<8)(\mathrm{n}=109)$ & $\mathrm{p}$-value \\
\hline \multicolumn{5}{|l|}{ Demographic characteristics } \\
\hline Age (y) & $72.96 \pm 6.41$ & $73.10 \pm 6.54$ & $72.89 \pm 6.36$ & 0.84 \\
\hline Sex, female & $112(67.5)$ & $48(84.2)$ & $64(58.7)$ & $<0.01^{*}$ \\
\hline $\operatorname{BMI}\left(\mathrm{kg} / \mathrm{m}^{2}\right)$ & $28.57 \pm 5.22$ & $29.54 \pm 5.63$ & $28.06 \pm 4.95$ & 0.08 \\
\hline Education (y) & $7.42 \pm 4.92$ & $5.33 \pm 4.60$ & $8.53 \pm 4.74$ & $<0.01^{*}$ \\
\hline Number of medications & $5.04 \pm 2.87$ & $6.05 \pm 3.13$ & $4.51 \pm 2.59$ & $<0.01^{*}$ \\
\hline Falls & $54(32.5)$ & $25(43.9)$ & $29(26.6)$ & $0.03^{*}$ \\
\hline MMSE score & $26.49 \pm 3.69$ & $26.13 \pm 3.80$ & $26.72 \pm 3.62$ & 0.45 \\
\hline \multicolumn{5}{|l|}{ Laboratory values } \\
\hline Hemoglobin (g/dL) & $12.77 \pm 1.28$ & $12.39 \pm 1.17$ & $12.97 \pm 1.30$ & $<0.01^{*}$ \\
\hline CRP & $4.50 \pm 7.36$ & $4.95 \pm 8.67$ & $4.19 \pm 6.34$ & 0.36 \\
\hline Albumin & $4.14 \pm 0.33$ & $4.08 \pm 0.35$ & $4.17 \pm 0.31$ & 0.16 \\
\hline Folic acid & $9.89 \pm 4.47$ & $9.50 \pm 5.08$ & $10.10 \pm 4.11$ & 0.12 \\
\hline Vitamin D (ng/mL) & $24.13 \pm 10.96$ & $21.68 \pm 9.33$ & $25.48 \pm 11.58$ & $0.03^{*}$ \\
\hline Vitamin B12 (pg/mL) & $389.32 \pm 246.09$ & $335.38 \pm 190.76$ & $418.10 \pm 267.44$ & $0.04^{*}$ \\
\hline Blood Glucose (mg/dL) & $111.39 \pm 40.26$ & $119.14 \pm 53.35$ & $107.26 \pm 30.64$ & 0.12 \\
\hline $\mathrm{TSH}(\mathrm{mLU} / \mathrm{L})$ & $1.80 \pm 1.54$ & $1.96 \pm 1.79$ & $1.72 \pm 1.39$ & 0.33 \\
\hline \multicolumn{5}{|l|}{ Comorbidities } \\
\hline Cerebrovascular disease & $8(4.8)$ & $4(7.0)$ & $4(3.7)$ & 0.44 \\
\hline Diabetes mellitus & $55(33.1)$ & $20(35.1)$ & $35(32.1)$ & 0.73 \\
\hline Hypertension & $109(65.7)$ & $38(66.7)$ & $71(65.1)$ & 0.86 \\
\hline Hyperlipidemia & $44(26.5)$ & $19(33.3)$ & $25(22.9)$ & 0.19 \\
\hline Congestive cardiac failure & $8(4.8)$ & $4(7.0)$ & $4(3.7)$ & 0.44 \\
\hline COPD & $17(10.2)$ & $5(8.8)$ & $12(11.0)$ & 0.79 \\
\hline Dementia & $18(10.8)$ & $6(10.5)$ & $12(11.0)$ & 0.92 \\
\hline Sarcopenia & $15(9.0)$ & $6(10.5)$ & $9(8.3)$ & 0.62 \\
\hline \multicolumn{5}{|c|}{ Comprehensive geriatric assessment } \\
\hline Fried frailty score & $1.56 \pm 1.30$ & $2.15 \pm 1.41$ & $1.24 \pm 1.12$ & $<0.01^{*}$ \\
\hline Fried frailty states & & & & $0.01^{*}$ \\
\hline No abnormalities & $37(22.3)$ & $7(12.3)$ & $30(27.5)$ & \\
\hline Pre-frail & $94(56.6)$ & $29(50.9)$ & $65(59.6)$ & \\
\hline Frail & $35(21.1)$ & $21(36.8)$ & $14(12.8)$ & \\
\hline POMA total score & $25.68 \pm 3.44$ & $24.26 \pm 3.96$ & $26.42 \pm 2.88$ & $<0.01^{*}$ \\
\hline POMA balance score & $14.55 \pm 2.15$ & $13.61 \pm 2,5$ & $15.05 \pm 1.75$ & $<0.01^{*}$ \\
\hline POMA gait score & $11.13 \pm 1.51$ & $10.65 \pm 1.70$ & $11.39 \pm 1.34$ & $<0.01^{*}$ \\
\hline IADL score & $19.59 \pm 4.59$ & $18.42 \pm 5.31$ & $20.20 \pm 4.06$ & $0.01^{*}$ \\
\hline BADL score & $92.78 \pm 8.41$ & $89.16 \pm 10.82$ & $94.68 \pm 6.07$ & $<0.01^{*}$ \\
\hline GDS score & $3.32 \pm 3.63$ & $5.91 \pm 3.9$ & $2.04 \pm 2.63$ & $<0.01^{*}$ \\
\hline HADS-A score & $6.66 \pm 4.77$ & $9.52 \pm 4.28$ & $5.16 \pm 4.32$ & $<0.01^{*}$ \\
\hline MNA score & $12.84 \pm 1.56$ & $12.16 \pm 1.78$ & $13.19 \pm 1.30$ & $<0.01^{*}$ \\
\hline Handgrip strength (kg) & $19.37 \pm 9.46$ & $15.37 \pm 8.64$ & $22.95 \pm 9.37$ & $<0.01^{*}$ \\
\hline Low muscle strength & $76(45.8)$ & $33(57.9)$ & $43(39.4)$ & $<0.01^{*}$ \\
\hline $\mathrm{SMM}(\mathrm{kg})$ & $18.69 \pm 4.53$ & $17.14 \pm 3.62$ & $19.50 \pm 4.76$ & $<0.01^{*}$ \\
\hline $\operatorname{SMI}\left(\mathrm{kg} / \mathrm{m}^{2}\right)$ & $7.45 \pm 1.31$ & $7.15 \pm 1.23$ & $7.60 \pm 1.32$ & $0.03^{*}$ \\
\hline Low muscle quantity & $27(16.3)$ & $9(15.8)$ & $18(16.5)$ & 0.90 \\
\hline Gait speed (m/s) & $1.00 \pm 0.34$ & $0.85 \pm 032$ & $1.08 \pm 0.32$ & $<0.01^{*}$ \\
\hline Low physical performance & $48(28.9)$ & $26(45.6)$ & $22(20.2)$ & $<0.01^{*}$ \\
\hline $\mathrm{BMR}(\mathrm{kcal})$ & $1,408.96 \pm 215.13$ & $1,360.87 \pm 210.27$ & $1,434.11 \pm 214.30$ & $0.03^{*}$ \\
\hline
\end{tabular}

Values are expressed as mean (standard deviation) for continuous variables and percent was reported for categorical variables.

BMI, body mass index; MMSE, Mini-Mental State Examination; TSH, thyroid-stimulating hormone; COPD, chronic obstructive pulmonary disease; TFI, Tilburg Frailty Indicator; POMA, Performance-Oriented Movement Association; BADL, basic activities of daily living; IADL, instrumental activities of daily living; CDR, Clinical Dementia Rating Score; GDS, Geriatric Depression Scale; HADS-A, Hospital Anxiety and Depression Scale-Anxiety; MNA, Mini Nutritional Assessment; SMM, skeletal muscle mass; SMI, skeletal muscle mass index; BMR, basal metabolic rate. ${ }^{*} \mathrm{p}<0.05$. 
nent frailty assessment tools. ${ }^{7)}$ The TFI is a multidimensional, useful, and fast instrument to assess the physical, psychological, and social components of frailty in older adults. ${ }^{8)}$ Fried frailty is the most widely used scale for identifying frailty. The scale has five criteria-weight loss, exhaustion, low physical activity, slowness, and weakness. According to this scale, a score $>3$ or more indicated frailty. ${ }^{5)}$ A cutoff score was calculated for the TFI based on the Fried Frailty, which is considered the "gold standard" for evaluating frailty. ${ }^{24)}$ We found that a TFI cutoff score of 8 showed the optimal specificity and sensitivity values. Gobbens et al. ${ }^{8)}$ reported a TFI cutoff score of 5 to identify frailty. Our cutoff score was 3 points

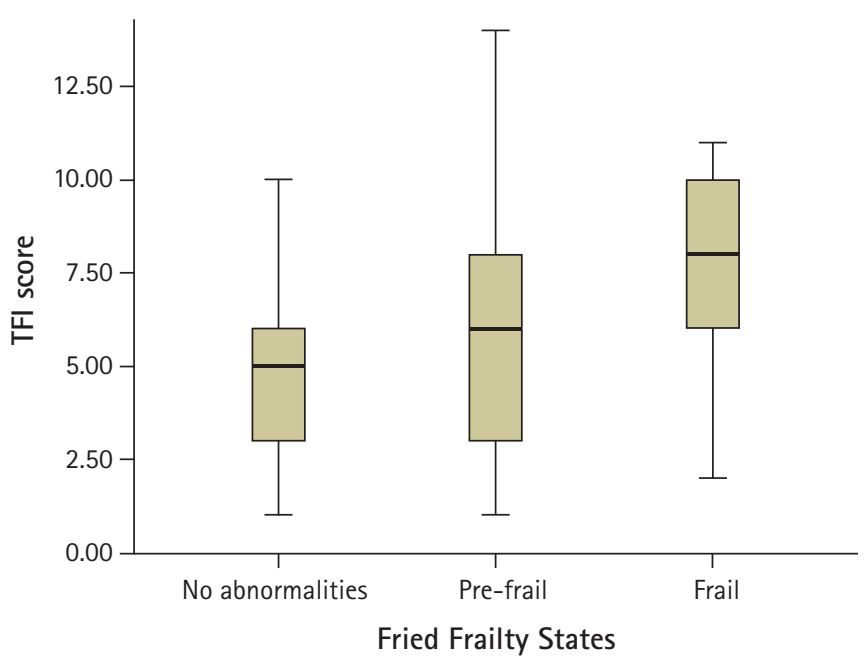

Fig. 1. Box-plot of the frailty of the Tilburg Frailty Indicator (TFI) score. higher than that of Gobbens' study. Gobbens' study included community-dwelling individuals aged $\geq 75$ years, with a mean participant age of 80 years. ${ }^{8)}$ Thus, the cutoff score may have differed due to the difference in mean age in these studies. ${ }^{8)}$ In addition, factors such as ethnicity and comorbidities can also affect the cutoff score. $^{9,11)}$

Among the many subjective and objective frailty assessment methods, ${ }^{26)}$ Fried Frailty is commonly used. ${ }^{5,27)}$ This instrument classifies older adults as frail, pre-frail, or non-frail, based on five criteria. ${ }^{5)}$ While Fried Frailty predominantly assesses physical frail-

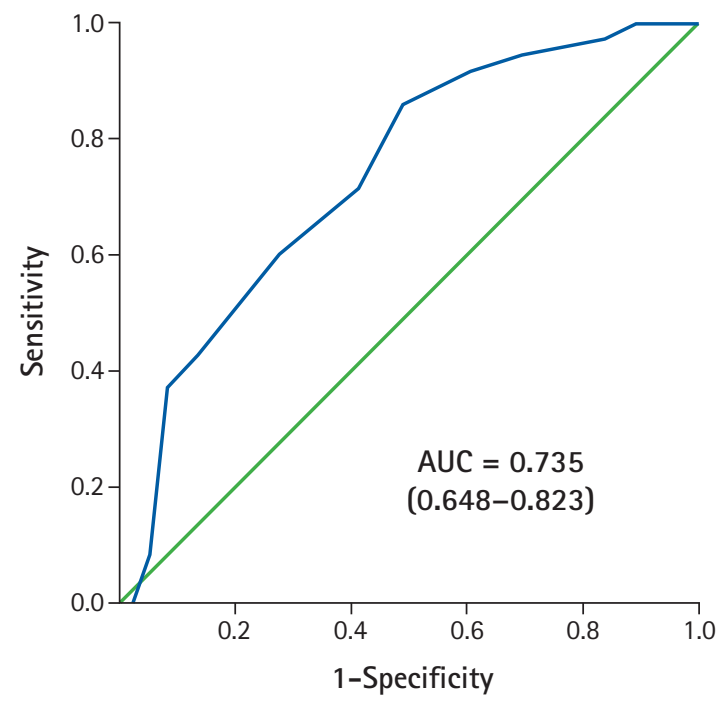

Fig. 2. Receiver operating characteristic curve for the Tilburg Frailty Indicator (TFI) cutoff value. AUC, area under the curve.

Table 2. Binary logistic regression model for frailty

\begin{tabular}{|c|c|c|c|c|c|c|c|c|}
\hline \multirow{2}{*}{ Variable } & \multicolumn{4}{|c|}{ TFI score $\geq 8$ (frail) } & \multicolumn{4}{|c|}{ Fried score $\geq 3$ (frail) } \\
\hline & B & SE & $\mathrm{p}$-value & OR $(95 \% \mathrm{CI})$ & B & SE & p-value & OR $(95 \% \mathrm{CI})$ \\
\hline POMA total & -0.15 & 0.06 & $0.01^{*}$ & $0.85(0.75-0.96)$ & -0.18 & 0.06 & $<0.01^{*}$ & $0.82(0.72-0.94)$ \\
\hline POMA gait & -0.21 & 0.12 & 0.09 & $0.80(0.62-1.03)$ & -0.18 & 0.13 & 0.15 & $0.83(0.64-1.07)$ \\
\hline POMA balance & -0.30 & 0.11 & $<0.01^{*}$ & $0.73(0.59-0.91)$ & -0.41 & 0.11 & $<0.01^{*}$ & $0.66(0.52-0.83)$ \\
\hline Walking speed & -1.41 & 0.73 & 0.05 & $0.24(0.05-1.02)$ & -5.82 & 1.30 & $<0.01^{*}$ & $0.01(0.01-0.03)$ \\
\hline BADL & -0.08 & 0.02 & $<0.01^{*}$ & $0.91(0.86-0.96)$ & -0.08 & 0.03 & $<0.01^{*}$ & $0.91(0.86-0.97)$ \\
\hline HADS-A & 0.19 & 0.04 & $<0.01^{*}$ & $1.21(1.11-1.33)$ & 0.06 & 0.04 & 0.18 & $1.06(0.97-1.16)$ \\
\hline GDS & 0.30 & 0.07 & $<0.01^{*}$ & $1.35(1.17-1.56)$ & 0.13 & 0.06 & 0.05 & $1.13(1.00-1.29)$ \\
\hline IADL & -0.06 & 0.04 & 0.14 & $0.93(0.85-1.02)$ & -0.15 & 0.05 & $<0.01^{*}$ & $0.85(0.77-0.95)$ \\
\hline MNA & -0.40 & 0.12 & $<0.01^{*}$ & $0.66(0.52-0.85)$ & -0.34 & 0.13 & $0.01^{*}$ & $0.70(0.54-0.92)$ \\
\hline Low MS & 1.10 & 0.41 & $<0.01^{*}$ & $3.03(1.35-6.78)$ & 1.39 & 0.46 & $<0.01^{*}$ & $4.03(1.61-10.10)$ \\
\hline Low SM & 0.21 & 0.51 & 0.67 & $1.24(0.45-3.42)$ & -0.60 & 0.60 & 0.31 & $0.54(0.16-1.79)$ \\
\hline Sarcopenia & 0.34 & 0.64 & 0.58 & $1.41(0.40-5.00)$ & 0.22 & 0.67 & 0.74 & $1.25(0.33-4.68)$ \\
\hline
\end{tabular}

TFI, Tilburg Frailty Indicator; GDS, Geriatric Depression Scale; POMA, Performance-Oriented Movement Association; BADL, basic activities of daily living; HADS-A, Hospital Anxiety and Depression Scale-Anxiety; MS, muscle strength; SM, skeletal muscle; SE, standard error; OR, odds ratio; CI, confidence interval.

${ }^{*} \mathrm{p}<0.05$. 
ty, it also has good validity for assessing frailty. ${ }^{5,28,29)}$ We found that the Fried score was more closely related to physical parameters, while the TFI score was more associated with both psychosocial and physical parameters. Thus, the TFI is multidimensional and more comprehensive in evaluating frailty. However, more studies on this subject are required.

Frailty is an important problem that affects the physical and psychological performance of older adults. ${ }^{2)}$ Physical and psychological parameters decrease in frail older adults compared to non-fragile ones. ${ }^{2,3)}$ Consistent with the literature, we found better measures of psychosocial and physical parameters in the non-frail group compared to those in the frail group. Together, these results show the importance of holistic approaches toward frail older adults.

Frailty and sarcopenia are important age-related concerns, with overlap between the two conditions. Frailty and sarcopenia are similar, especially in terms of evaluating physical parameters. ${ }^{30)}$ Sarcopenia is now formally recognized as a muscle disease that occurs in older adults but that can also occur earlier in life. ${ }^{20)}$ The EWGSOP-2 recommends assessments of muscle strength, muscle quantity, and physical performance in the evaluation of sarcopenia. ${ }^{20)}$ We found that neither the TFI nor the Fried scores were associated with sarcopenia, while frailty was associated with low muscle strength, which is the sub-evaluation parameter of sarcopenia. Thus, frailty and sarcopenia are different concepts, between which only physical parameters can be similar. ${ }^{30)}$

This study has some limitations. First, the cross-sectional design precluded inferences about the direction of causality among the variables. Second, BIA was used to assess muscle mass. While it is a valid tool for assessing muscle mass, it does not measure mass directly.

In conclusion, the results of this study suggested an optimal TFI cutoff score of 8 as a frailty instrument in community-dwelling older adults. Additionally, the TFI included physical, psychologi$\mathrm{cal}$, and social aspects, thereby providing a multidimensional evaluation of frailty.

\section{ACKNOWLEDGMENTS}

\section{CONFLICT OF INTEREST}

The researchers claim no conflicts of interest.

\section{FUNDING}

None.

\section{AUTHOR CONTRIBUTIONS}

Conceptualization, GO, EAB, BG, NI, ATI; Data curation, GO,
EAB; Funding acquisition, GO, EAB, BG, NI, ATI; Investigation, GO, EAB, BG; Methodology, GO, EAB, BG, NI, ATI; Project administration, GO, NI, ATI; Supervision, NI, ATI; Writing-original draft, GO, EAB, BG; Writing-review \& editing, GO, EAB, BG, NI, ATI.

\section{REFERENCES}

1. Cesari M. Physical frailty and sarcopenia: development of a framework for supporting interventions against incident mobility disability. Ann Geriat Med Res 2017;21:42-8.

2. Gobbens RJ, Luijkx KG, Wijnen-Sponselee MT, Schols JM. In search of an integral conceptual definition of frailty: opinions of experts. J Am Med Dir Assoc 2010;11:338-43.

3. Gobbens RJ, Luijkx KG, Wijnen-Sponselee MT, Schols JM. Toward a conceptual definition of frail community dwelling older people. Nurs Outlook 2010;58:76-86.

4. Gobbens RJ, Schols JM, van Assen MA. Exploring the efficiency of the Tilburg Frailty Indicator: a review. Clin Interv Aging 2017;12:1739-52.

5. Fried LP, Tangen CM, Walston J, Newman AB, Hirsch C, Gottdiener J, et al. Frailty in older adults: evidence for a phenotype.J Gerontol A Biol Sci Med Sci 2001;56:M146-56.

6. Abellan van Kan G, Rolland YM, Morley JE, Vellas B. Frailty: toward a clinical definition. J Am Med Dir Assoc 2008;9:71-2.

7. Sutton JL, Gould RL, Daley S, Coulson MC, Ward EV, Butler AM, et al. Psychometric properties of multicomponent tools designed to assess frailty in older adults: a systematic review. BMC Geriatr 2016;16:55.

8. Gobbens RJ, van Assen MA, Luijkx KG, Wijnen-Sponselee MT, Schols JM. The Tilburg Frailty Indicator: psychometric properties. J Am Med Dir Assoc 2010;11:344-55.

9. Dong L, Liu N, Tian X, Qiao X, Gobbens RJ, Kane RL, et al. Reliability and validity of the Tilburg Frailty Indicator (TFI) among Chinese community-dwelling older people. Arch Gerontol Geriatr 2017;73:21-8.

10. Topcu Y, Tufan F, Kilic C. Turkish version of the Tilburg Frailty Indicator. Clin Interv Aging 2019;14:615-20.

11. Coelho T, Santos R, Paul C, Gobbens RJ, Fernandes L. Portuguese version of the Tilburg Frailty Indicator: transcultural adaptation and psychometric validation. Geriatr Gerontol Int 2015; 15:951-60

12. Ates Bulut E, Soysal P, Isik AT. Frequency and coincidence of geriatric syndromes according to age groups: single-center experience in Turkey between 2013 and 2017. Clin Interv Aging 2018;13:1899-905.

13. American Psychiatric Association. Diagnostic and statistical 
manual of mental disorders: DSM-5. Arlington, VA: American Psychiatric Association; 2013.

14. Gungen C, Ertan T, Eker E, Yasar R, Engin F. [Reliability and validity of the standardized Mini Mental State Examination in the diagnosis of mild dementia in Turkish population]. Turk Psikiyatri Derg 2002;13:273-81.

15. Ertan T, Eker E. Reliability, validity, and factor structure of the geriatric depression scale in Turkish elderly: are there different factor structures for different cultures? Int Psychogeriatr 2000; 12:163-72.

16. Katz S. Assessing self-maintenance: activities of daily living, mobility, and instrumental activities of daily living. J Am Geriatr Soc $1983 ; 31: 721-7$.

17. Tinetti ME. Performance-oriented assessment of mobility problems in elderly patients. J Am Geriatr Soc 1986;34:119-26.

18.Guigoz Y. The Mini Nutritional Assessment (MNA) review of the literature: what does it tell us? J Nutr Health Aging 2006; 10:466-87.

19. Zigmond AS, Snaith RP. The hospital anxiety and depression scale. Acta Psychiatr Scand 1983;67:361-70.

20. Cruz-Jentoft AJ, Bahat G, Bauer J, Boirie Y, Bruyere O, Cederholm T, et al. Sarcopenia: revised European consensus on definition and diagnosis. Age Ageing 2019;13:273-81.

21. Ates Bulut E, Soysal P, Dokuzlar O, Kocyigit SE, Aydin AE, Yavuz I, et al. Validation of population-based cutoffs for low muscle mass and strength in a population of Turkish elderly adults. Aging Clin Exp Res 2020;32:1749-55.
22. Janssen I, Heymsfield SB, Baumgartner RN, Ross R. Estimation of skeletal muscle mass by bioelectrical impedance analysis. J Appl Physiol (1985) 2000;89:465-71.

23. Bujang MA, Adnan TH. Requirements for minimum sample size for sensitivity and specificity analysis. J Clin Diagn Res 2016;10:YE01-YE06.

24. Toosizadeh N, Wendel C, Hsu CH, Zamrini E, Mohler J. Frailty assessment in older adults using upper-extremity function: index development. BMC Geriatr 2017;17:117.

25. Swets JA. Measuring the accuracy of diagnostic systems. Science 1988;240:1285-93.

26. Bouillon K, Kivimaki M, Hamer M, Sabia S, Fransson EI, Singh-Manoux A, et al. Measures of frailty in population-based studies: an overview. BMC Geriatr 2013;13:64.

27. Chang SF, Lin PL. Frail phenotype and mortality prediction: a systematic review and meta-analysis of prospective cohort studies. Int J Nurs Stud 2015;52:1362-74.

28. Avila-Funes JA, Medina-Campos RH, Tamez-Rivera O, Navarrete-Reyes AP, Amieva H, Aguilar-Navarro S. Frailty is associated with disability and recent hospitalization in community-dwelling elderly: the Coyoacan cohort. J Frailty Aging 2014;3:206-10.

29. Rockwood K, Andrew M, Mitnitski A. A comparison of two approaches to measuring frailty in elderly people. J Gerontol A Biol Sci Med Sci 2007;62:738-43.

30. Cesari M, Landi F, Vellas B, Bernabei R, Marzetti E. Sarcopenia and physical frailty: two sides of the same coin. Front Aging Neurosci 2014;6:192. 